\title{
USO DE MÉTODOS PARTICIPATIVOS EN EL DESARROLLO DE UN PROGRAMA DE EDUCACIÓN PARA EL AUTOCUIDADO DE LA DIABETES EN ADULTOS MAYORES EN CANADÁ
}

\author{
Pilar Camargo-Plazas ${ }^{1}$, Idevania Costa ${ }^{2}$, Lenora Duhn ${ }^{1}$, \\ Beatriz Alvarado ${ }^{3}$ y Deborah Tregunno ${ }^{1}$ \\ 1,3 School of Nursing, Queen's University, Ontario, Canadá. mdpc@queensu.ca; duhnı@queensu.ca; alvaradb@queensu.ca \\ tregunno@queensu.ca \\ ${ }^{2}$ School of Nursing, Lakehead University, Ontario, Canadá. igcosta@lakeheadu.ca
}

\begin{abstract}
Resumen. El presente trabajo presenta el uso de métodos participativos para desarrollar un programa de educación para el autocuidado de la diabetes en adultos mayores en Canadá Objetivos; presentar nuestra experiencia con el uso de métodos participativos con una comunidad de adultos mayores con diabetes. Métodos: Para este estudio se uso una metodología investigación-acción participativa en una comunidad rural de Ontario, Canadá. La implementación de comité asesor, el uso del modelo de salud publica PRECEDER-PROCEDER es presentado, así como el uso del World café o café del munod, finalmente se presenta el método de fotovoz que será usado con la comunidad. Conclusiones. El uso de métodos participativos ha sido un proceso enriquecedor en donde le hemos dado voz a la comunidad para que guie el proyecto. Asimismo, hemos aprendido a conocer las necesidades de la población de adultos mayores en esta comunidad. Igualmente hemos aprendido que el uso de métodos participativos debe estar basado en el contexto en donde la investigación toma lugar.
\end{abstract}

Palabras clave: Adultos Mayores; Diabetes; Autocuidado; Investigación-Acción Participativa; Determinantes Sociales de la Salud.

\section{USING PARTICIPATORY METHODS IN THE DEVELOPMENT OF DIABETES SELF-CARE MANAGEMENT EDUCATIONAL PROGRAMS FOR SENIORS: PRELIMINARY FINDINGS}

\begin{abstract}
The following paper presents preliminary findings of a Participatory Action Research (PAR) project aimed to engage seniors in the development, design, implementation, and evaluation of an educational program in self-management for seniors with diabetes. Methods: Using PAR, we will engage seniors with diabetes in understanding their experiences with diabetes selfmanagement through the implementation of participatory processes in a rural community in Ontario, Canada. We present our experiences engaging community members in our advisory committee, using the PRECEDE-PROCEED model to assess the community needs and planning the implementation of a World café and photovoice participatory methods. Conclusion: Identifying the factors and barriers influencing education in self-management and determining the broader social determinants of health are critical to addressing social and health inequities for seniors. Among the factors that contribute to the suboptimal practice of self-care management in older adults, health literacy has been identified as an essential and potentially ameliorable issue. By using participatory methods of engagement, we will give voice to the unique needs of seniors with diabetes. We have also learned that the use of participatory methods must be based on the context in which the research takes place.
\end{abstract}

Keywords: Seniors; Diabetes; Self-care Management; Participatory Action Research; Social Determinants of Health.

\section{INTRODUCCIÓN}

A nivel global se calcula que cerca de 463 millones de adultos padecen de diabetes y se espera que para el 2045 este número se incremente a 700 millones de individuos(IDF, 
2019). En el 2016, 1.6 millones de personas fallecieron como resultado de la diabetes (WHO, 2019). Igualmente, cerca de 2.2 millones de las muertes por enfermedades cardiovasculares y enfermedades renales crónicas son consecuencia de complicaciones de la diabetes (WHO, 2019). En el Canadá cerca de 11 millones de individuos viven con diabetes o pre-diabetes. En la provincia de Ontario alrededor de 4.4 millones de personas viven con esta enfermedad (Diabetes Canada, 2018). Sin el tratamiento adecuado la diabetes conlleva a complicaciones y a una muerte prematura. Si no se controla con medicación y autocuidado, la hiperglicemia puede causar eventos cerebrovasculares, nefropatía, infarto, amputación, retinopatías y pie diabético (WHO, 2019). La diabetes y sus complicaciones generan grandes perdidas económicas para aquellos que viven con esa condición, sus familias, para los sistemas de salud y las economías nacionales, en forma de gastos médicos directos y por perdida de trabajo e ingresos (IDF, 2019; WHO, 2019). En el Canadá, el gasto en tratamiento de la diabetes se incremento de $\$ 14$ billones en el 2008 a $\$ 30$ billones en el 2019 (Diabetes Canadá, 2018). Si a esto se le suma que el 33\% de los canadienses que tienen diabetes pueden identificar menos de la mitad de los signos de alarma, entonces la morbilidad y discapacidad funcional resultantes de la diabetes son aspectos que pueden ser modificados con un buen autocuidado.

El autocuidado es importante en la reducción de complicaciones y en el mejoramiento de la calidad de vida de las personas que viven con diabetes. No obstante, para que sea efectivo el autocuidado la educación proveída a las personas con diabetes debe tener un enfoque interdisciplinario(Geboers et al., 2015; Harris, 2019). La educación en salud se considera un proceso interactivo en el cual las personas o las comunidades aprenden aspectos que favorecen la prevención, promoción, el mantenimiento o la restauración de la salud(Beck et al., 2019). Para que la educación en salud alcance su objetivo final de prevención y promoción de la salud se requiere que el profesional de salud tenga experiencia en el tema y sea un facilitador en el proceso de aprendizaje de sus pacientes de manera que estos se convierten en agentes activos que imparten el conocimiento aprendido dentro de sus comunidades. Sin embargo, estudios han mostrado como la educación para el autocuidado de la diabetes no ha sido un proceso interactivo si no mas bien de entrega unidireccional de información en donde el paciente con diabetes solo recibe instrucciones acerca de su enfermedad (Costa, Camargo-Plazas, \& Tregunno, 2017; Geboers et al., 2015; Harris, 2019). El manejo de la diabetes necesita que las personas que padecen esa condición tomen decisiones acertadas para el cuidado de su salud, para lo cual se necesita programas 
de educación en diabetes que enfaticen la participación activa y que considere las necesidades y el contexto único de cada individuo. Otro aspecto que se considera importante en el desarrollo de programas de educación para el autocuidado de la diabetes es el nivel de alfabetización en salud de las personas. La alfabetización en salud se define como la capacidad de una persona para encontrar, comprender y utilizar la información de manera adecuada y de esta manera acceder a los servicios de salud necesarios. De allí, que la alfabetización en salud este relacionada con el concepto de autonomía (Rootman \& Gordon-El-Bihbety, 2008).

Es por eso por lo que el autocuidado es fundamental en el manejo de las enfermedades crónicas para evitar complicaciones. No obstante, cerca de un $88 \%$ de los adultos mayores en el Canadá carecen de los conocimientos, habilidades y destrezas provenientes de una educación para la salud para controlar la enfermedad y prevenir complicaciones (Canadian Public Health Association, 2014). En la población canadiense se ha encontrado una asociación entre los niveles de alfabetización en salud y la prevalencia de diabetes en donde individuos con niveles bajos de alfabetización tienen mayor probabilidad de desarrollar diabetes (Canadian Council on Learning, 2008). Otros estudios han demostrado como los individuos con diabetes expresan frustración al tratar de aplicar, sin éxito, lo aprendido en las sesiones de educación a su vida diaria sin éxito, puesto que la aplicabilidad de los aprendido es siempre desafiada por factores contextuales como los bajos ingresos económicos, la falta de transporte, el desempleo, el acceso a los servicios de salud o a los tratamientos, el vivir en un área remota o rural y los riesgos ambientales (Agarwal et al., 2018; Al Sayah \& Williams, 2012). Es desde este contexto que nuestro trabajo participativo emerge para atender las necesidades únicas de los adultos mayores con diabetes. Nuestro plan es desarrollar un programa de educación para el autocuidado de la diabetes que incorpore las experiencias, el contexto único y los determinantes sociales de la salud de los adultos mayores del sureste de Ontario. El propósito de este artículo es describir como ha sido el proceso de emprender una investigación-acción participativa (IAP) con adultos mayores que viven con diabetes. Nuestro objetivo es presentar los resultados preliminares de nuestro trabajo que busca describir como ha sido el proceso de problematización del autocuidado en personas mayores con diabetes y que métodos pensamos implementar para delinear mejor las estrategias de educación para el autocuidado. Empezaremos presentando los objetivos del estudio y su contexto, continuaremos con una descripción de la metodología del estudio, nuestro plan del trabajo participativo hecho hasta el momento con 
sus ventajas y desafíos, finalizando con lo que hemos aprendido con este estudio y los planes futuros.

\section{El Estudio}

El objetivo de este estudio es desarrollar un programa de educación para el autocuidado de la diabetes en adultos mayores. Siguiendo los lineamientos de una IAP, adultos mayores, profesionales de la salud e investigadores formularemos el proceso de diseño, implementación y evaluación del programa de educación para la diabetes. Nuestra idea inicial era invitar un grupo de expertos en el tema para que le presentaran a miembros de la comunidad información acerca del autocuidado de la diabetes y los efectos de los determinantes sociales de la salud. Terminadas las presentaciones habíamos planeado usar los círculos de cultura descritos por Paulo Freire para así determinar las necesidades de autocuidado en la comunidad. Aunque el objetivo no ha cambiado los métodos de participación han evolucionado para incorporar las necesidades únicas de la comunidad de Sharbot Lake. Este trabajo de investigación ofrece la oportunidad para que los adultos mayores, los profesionales de la salud y los investigadores determinen la forma mas efectiva de atender a las necesidades de salud de los adultos mayores con diabetes. El proyecto se esta desarrollando en colaboración con un centro de salud comunitario ubicado en el municipio de Sharbot Lake, en la provincia de Ontario, Canadá. Según las estadísticas el número de adultos mayores que viven en esta región se ha duplicado entre los años 2006 y 2016. Igualmente, en esta región es común encontrar mayor exposición a condiciones socioeconómicas adversas que en otras regiones, lo cual ha contribuido a una incidencia mas alta de enfermedades crónicas y a la exposición continua a diversos factores de riesgo. La falta de transporte, acceso a los servicios de salud, la ruralidad, la pobreza y la falta de educación son factores que dificultan la creación de programas de autocuidado adecuados en la región.

Esta investigación fue aprobada por el comité de ética de Queen's University, en Kingston, Ontario. Tenemos planeado invitar 30 participantes de la comunidad, adultos mayores de 65 años y más. Las actividades de investigación desarrolladas y futuras son descritas en la siguiente sección. El análisis de datos es participativo y sigue un lineamiento temático (van Manen, 2014). Los determinantes sociales de la salud son un aspecto fundamental a tener en cuenta por esta razón nuestro estudio es guiado por el modelo teórico descrito por Loppie-Reading and Wien (2009) quienes clasificaron los determinantes sociales de la salud 
en tres diferentes categorías: (1) distal (ej. aspectos históricos, políticos, sociales y el contexto económico), (2) intermedios (ej. la infraestructura de la comunidad, recursos, sistemas y capacidades), y (3) proximales (ej. las conductas de salud, los aspectos físicos y del medio ambiente). Este modelo guiará el análisis de ideas y estrategias priorizadas durante el proceso de recolección de datos. Las actividades participativas son conducidas por el método de educación participativa descrito por Paulo Freire (2014). En las siguientes secciones se presenta en forma más detallada el uso de métodos participativos de este proyecto.

\section{METODOLOGÍA}

La IAP se conceptualiza como una metodología científica enfocada en un elemento clave: la participación activa de diferentes agentes en el proceso de investigación (Asirifi, 2019; Kidd, Davidson, Frederick, \& Kral, 2018). A diferencia de otras metodologías de investigación, en la IAP es la propia comunidad la encargada de definir el problema, crear soluciones y diseminar los resultados. La IAP ha surgido como respuesta a la forma clásica de intervenir, en la cual el investigador como único poseedor del conocimiento se encarga de definir las prioridades a investigar sin tener en cuenta a los destinatarios de los resultados (Akintobi et al., 2018; Kidd et al., 2018). Históricamente la investigación participativa esta vinculada a la movilización de sectores sociales vulnerables, promoviendo formas de hacer investigación cuyo conocimiento generado sea utilizado para el beneficio de la comunidad o grupo poblacional donde la investigación se realiza (Etowa, Bernard, Oyinsan, \& Clow, 2007). La investigación participativa promueve por una parte la expansión del conocimiento, y por otra genera respuestas concretas a problemas que plantea una comunidad o grupo poblacional con la alternativa de cambio o transformación (Paredes-Chi \& Castillo-Burguete, 2018). Investigadores que utilizan IAP tratan de redistribuir las relaciones jerárquicas del proceso de investigación para poder comprender de forma única los problemas sociales. El potencial de la IAP está centrado en la producción de conocimiento articulado de manera crítica, tanto de la ciencia como de la experiencia popular con el fin de reorientarlos hacia la creación de acciones transformadoras(Asirifi, 2019). En la IAP los miembros de la comunidad son coinvestigadores quienes junto a los investigadores abordan conjuntamente todas las etapas del proceso de investigación y de intervención social (Esienumoh, Allotey, \& Waterman, 2018; Kidd et al., 2018). De esta manera, en el diseño, desarrollo, implementación y evaluación de programas de salud no cabe duda que la sostenibilidad de cualquier estrategia de educación en salud descansa en la participación activa de los diversos actores 
en los procesos de decisiones. Como investigadoras en el área de la salud consideramos importante el uso de IAP para el desarrollo de programas educativos para el autocuidado de la diabetes que involucren de forma activa a los individuos que viven con esta enfermedad.

\subsection{Desarrollo de la Colaboración}

El proyecto de investigación presentado en este artículo esta basado en un trabajo previo de teoría fundamentada orientado a explicar el proceso de participación en autocuidado de personas con úlceras activas del pie diabético. Los resultados de este trabajo preliminar revelaron que los participantes tenían preparación limitada sobre el autocuidado de la diabetes y sus complicaciones (Costa et al., 2018). Basados en este hallazgo decidimos desarrollar un proyecto de investigación orientado a explorar la educación para el autocuidado de la diabetes en adultos mayores usando una metodología participativa. Nuestro primer contacto incluyo a miembros de la red local de servicios de salud del suroeste de Ontario. Fue a través de nuestra red local que logramos el contacto con un centro comunitario en la región Central de Frontenac en el municipio de Sharbot Lake. Allí conocimos y presentamos nuestra idea de examinar los programas de educación para el autocuidado de la diabetes a la directora ejecutiva del centro comunitario y a la directora de programas. Ellas expresaron entusiasmo en nuestra idea resaltando la importancia de garantizar que la educación para el autocuidado responda efectivamente a las necesidades individuales de la población de adultos mayores en el municipio de Sharbot Lake. En sus propias palabras, "necesitamos evaluar si nuestro programa de educación en diabetes esta realmente abordando las necesidades de las personas mayores con diabetes de nuestra comunidad." El proceso de participación requirió varios encuentros con diferentes actores del centro de salud presentando y socializando nuestro proyecto.

\subsection{Formación del Comité Asesor}

La IAP se basa en la colaboración entre los investigadores y las comunidades para poder alinear la agenda investigativa con las prioridades de la comunidad (Kidd et al., 2018). La colaboración con miembros de la comunidad debe basarse en los principios de mutuo respeto, reconocimiento, relevancia y reciprocidad (Asirifi, 2019). Para poder asegurarse que el proceso investigativo sigue estos principios se han creado los comités asesores. Los comités asesores ofrecen la oportunidad para dialogar acerca del proceso de planeamiento, organización, implementación, y evaluación del proceso investigativo (Cramer, Lazoritz, 
Shaffer, Palm, \& Ford, 2018; Pinto, Spector, Rahman, \& Gastolomendo, 2015). En nuestro estudio como parte del proceso participativo decidimos formar un comité asesor que se encargue de supervisar el proyecto y garantizar que la voz de las personas mayores con diabetes

este siempre al centro de todos los procesos investigativos. Nuestro comité asesor esta constituido por una líder comunitaria adulto mayor con diabetes, dos profesionales de la salud que trabajan en el programa de diabetes, la directora de programas de Sharbot Lake, una nutricionista que trabaja en la red local de servicios de salud del suroeste de Ontario y los investigadores. Desde nuestro primer encuentro el comité asesor ha insistido en el uso de métodos de participación que propicien el diálogo entre la comunidad sobre temas sensibles como empleo, educación, transporte, apoyo social, acceso a los servicios de salud y sociales, acceso a tratamiento y adecuada alimentación. Nuestra idea inicial de invitar expertos en educación en salud y diabetes para luego en la sesión de la tarde implementar grupos focales fue cambiada por miembros del comité asesor por no ser suficientemente participativa. Nuestro comité asesor determinó que la meta final de la consulta con la comunidad debe ser la identificación de las necesidades de educación para el autocuidado de la diabetes de las personas mayores en el municipio de Sharbot Lake. Asimismo, se vio la necesidad de hacer una valoración de la comunidad para poder planear de forma mas adecuada nuestra consulta con la comunidad.

\subsection{Fase de Diagnostico Uso del Modelo PRECEDER-PROCEDER}

EI modelo PRECEDER-PROCEDER fue desarrollado por Lawrence W Green en 1974 para la planificación de programas y el desarrollo de intervenciones de salud pública (Castellanos \& Abrahamsen, 2014; Sezgin \& Esin, 2018). Este modelo proporciona una estructura integral para evaluar las necesidades de salud y la calidad de vida para así diseñar, implementar y evaluar programas de promoción de la salud(Shanta Bridges et al., 2018). El modelo PRECEDER-PROCEDER usa un método participativo desde la fase inicial hasta la final. En la fase PRECEDER se hace una valoración exhaustiva y detallada de todos los aspectos sociales, conductas, epidemiologia, administración, políticas de salud y la educación para así poder determinar las prioridades de trabajo y desarrollar la siguiente fase o PROCEDER que incluye las fases de intervención y evaluación(Nomura et al., 2019). En nuestro proyecto involucramos a dos estudiantes de ultimo año del programa de enfermería quienes como requisito de la clase NURS 405-Práctica en la Promoción de la Salud Comunitaria deben 
aplicar el modelo PRECEDER-PROCEDER. Como tutores de práctica guiamos a los estudiantes durante todo el proceso de implementación del modelo. En la fase PRECEDER los estudiantes entrevistaron unos pocos adultos mayores y profesionales de la salud que trabajan en educación de la diabetes para entender las necesidades de educación en diabetes, información que fue después corroborada con literatura en el tema. Los resultados de esta fase confirmaron que los programas de educación en autocuidado necesitan de la participación de los adultos mayores. Igualmente confirmaron la importancia de implementar un programa de educación que tenga en cuenta los determinantes sociales de la salud y que se ajuste a los niveles de alfabetización de la comunidad. En la segunda fase del modelo o PROCEDER los estudiantes crearon una serie de preguntas para ser usadas con la comunidad. La implementación de este modelo nos ayudo a darnos una idea de los problemas de la comunidad de Sharbot Lake. Con esas preguntas listas comenzamos a planear el uso de ellas usando la técnica participativa de café del mundo.

\subsection{World Café o Café del Mundo como Método Participativo}

El "world café" o café del mundo es una técnica participativa en donde un grupo de 4 o 5 personas conversan sobre un tema que les interesa o algún trabajo que piensan hacer juntos(Johnson et al., 2018). Es un método ideal para descubrir lo que la comunidad piensa y siente acerca de un tema. Después de la primera conversación, alguien se queda en la mesa como anfitrión mientras que los otros participantes se mueven de la mesa para retomar conversaciones previas en otras mesas (Dawkins \& Solomon, 2017). De esta manera, los temas de las distintas conversaciones se entrelazan y todos los participantes crean un dialogo colaborativo alrededor de asuntos que importan (Dawkins \& Solomon, 2017; O'Connor \& Cotrel-Gibbons, 2017). Ser anfitrión de un café del mundo no es difícil; sin embargo, se deben seguir unos lineamientos. Por ejemplo, se debe establecer el contexto para así determinar el propósito y los parámetros de la reunión; crear un espacio acogedor; explorar preguntas que son relevantes para la comunidad; alentar la contribución de todos; conectar diversas perspectivas; escuchar juntos patrones y percepciones; y el compartir descubrimientos colectivos (O'Connor \& Cotrel-Gibbons, 2017). La idea de usar este método igualmente se consultó con nuestro comité asesor. El plan era organizar un café del mundo con las preguntas generadas del trabajo de los estudiantes para así determinar las necesidades de educación de los adultos mayores con diabetes en Sharbot Lake. Sin embargo, después de una evaluación cuidadosa, el comité asesor decidió que, debido al nivel de alfabetización en salud de la comunidad y para evitar que los participantes se 
sientan juzgados o criticados por sus conductas o creencias en el autocuidado, se debería crear otra estrategia diferente al café del mundo para lograr la participación activa de los adultos mayores con diabetes.

\subsection{Uso de Fotovoz como Método Participativo}

La fotovoz es un método participativo que usa los recursos visuales y discursivos para el conocimiento de la realidad social (Budig et al., 2018; Newman, 2010). La fotovoz es un método orientado hacia el cambio social y el empoderamiento de los sujetos participantes (Twyman, Lehna, Fahey, Coty, \& Wishnia, 2015). Con esta técnica de investigación social se combina el uso de la fotografía con la acción comunitaria desde una perspectiva participativa (Budig et al., 2018; Twyman et al., 2015). A través de la fotovoz las personas pueden identificar, representar y mejorar sus comunidades por medio de una técnica fotográfica especifica. A través de las fotos se espera que las personas expresen sus ideas, concepciones, pensamientos, relaciones e interacciones favoreciendo así la implicación directa de los participantes en la producción de información visual (Twyman et al., 2015). El objetivo de la fotovoz es: a) permitir que las personas registren y reflejen las preocupaciones y realidades de sus comunidades; b) promover el dialogo crítico y el conocimiento de la comunidad a través de la discusión grupal sobre las fotografías producidas; y c) generar cambio en la comunidad afectada (Budig et al., 2018). Nuestra idea es invitar a adultos mayores de la comunidad con diabetes y prediabetes para que a través de la fotografía identifiquen aspectos específicos de su experiencia de vivir con diabetes, su contexto y las condiciones de vida que afectan el proceso de autocuidado de su enfermedad. La fotovoz será el método participativo que usaremos para entender las experiencias de vida de los adultos mayores de Sharbot Lake. Este método tiene el potencial de crear un espacio de dialogo en donde adultos mayores puedan presentar sus experiencias de autocuidado. En la siguiente sección presentamos como planeamos implementar esta técnica. Para implementar esta actividad tenemos las siguientes actividades planeadas:

a) Reunión inicial con miembros de la comunidad para conceptualizar el tema de la educación para el autocuidado de la diabetes. En esta fase nos reunimos con miembros de la comunidad para definir el objetivo de la fotovoz, concertar el tipo de fotografías que se tomaran, el tiempo para tomarlas y las anotaciones que pueden hacer una vez tomen las fotos. 
b) Después invitaremos a cada participante a una entrevista individual en donde ellos describirán donde se tomaron las fotografías y el significado de cada una de ellas. Se invitará luego al adulto mayor a seleccionar dos fotos para presentarle al grupo.

c) Invitaremos a los participantes a una actividad con grupos focales en donde cada individuo presentará las dos fotografías seleccionadas y compartirá el significado de cada foto. Luego el grupo creara a partir de ese diálogo una lista con las necesidades identificadas en las presentaciones. Asimismo, los participantes organizarán las prioridades identificadas de mayor a menor impacto, colocando de primeras aquellas necesidades que afectan su autocuidado de una forma más profunda. Los participantes dibujarán un árbol en donde el tronco representa el problema específico al que el grupo considera el problema mayor, las hojas mostraran las consecuencias del problema, y la raíz representará la causa del problema.

d) Se invitará a los participantes a crear una presentación corta para la comunidad, los profesionales de la salud y los investigadores en donde se muestren las fotografías seleccionadas con la presentación del árbol que describe el problema identificado por la comunidad en los grupos focales.

e) A raíz del dialogo se invitarán miembros de la comunidad a trabajar en conjunto con los investigadores en el desarrollo de otros proyectos de investigación orientados a mover a la acción las soluciones presentadas en la exhibición de fotografía.

\section{CONCLUSIÓN}

El uso de métodos participativos para el desarrollo de este proyecto ha sido exitoso en el desarrollo de estrategias participativas con adultos mayores. El tener un comité asesor ha abierto un espacio para que la reflexión y la concientización afloren y se consoliden durante el proceso participativo. Los métodos participativos se conocen por ayudar a las poblaciones más vulnerables a transformar las experiencias de vida, los pensamientos y los sentimientos relacionados a la educación para el autocuidado de la diabetes en adultos mayores. Existe una gran necesidad de identificar problemas sistémicos, locales y culturales que afectan a los adultos mayores con diabetes. Los métodos participativos brindan un lugar para la reflexión y creación de propuestas educativas que mejoren el autocuidado de la diabetes. Por lo tanto, el desarrollo de un programa de educación para el autocuidado de la diabetes debe comenzar con una visión ampliada de las experiencias vividas por los adultos mayores. El uso de métodos participativos facilita la colaboración mutua entre personas mayores, proveedores de servicios de salud e investigadores. Este método participativo de educación con datos experimentales y discusión grupal podría involucrar a las personas mayores en un proceso que transforma a los individuos en maestros emergentes y actores 
sociales en sus comunidades. Este documento ha tratado de ilustrar cómo los proveedores de salud y los educadores pueden asociarse con personas mayores que viven con diabetes de maneras que las interacciones sean mutuamente beneficiosas para mejorar su calidad de vida y evitar complicaciones. Este trabajo tiene el potencial de cambiar los programas tradicionales de educación para el autocuidado de la diabetes a una educación que incorpora el esfuerzo, la colaboración y transformación de los adultos mayores como agentes activos de cambio y decisión en el proceso investigativo.

Agradecimientos. Este trabajo ha sido financiado por el Canadian Institute of Health Research (ClHR)-Institute of Aging.

\section{REFERENCIAS}

Agarwal, G., Habing, K., Pirrie, M., Angeles, R., Marzanek, F. \& Parascandalo, J. (2018). Assessing health literacy among older adults living in subsidized housing: A cross-sectional study. Canadian Journal of Public Health, 109, 401-9.

Akintobi, T. H., Lockamy, E., Goodin, L., Hernandez, N. D., Slocumb, T., Blumenthal, D., . . . Hoffman, L. (2018). Processes and Outcomes of a Community-Based Participatory Research-Driven Health Needs Assessment: A Tool for Moving Health Disparity Reporting to Evidence-Based Action. Prog Community Health Partnersh, 12(1S), 139-147. doi:10.1353/cpr.2018.0029

Al Sayah, F. \& Williams, B. (2012). An integrated model of health literacy using diabetes as an exemplar. Canadian Journal of Diabetes, 36, 27-31.

Asirifi, M. A. (2019). Reflecting on Leadership Development through Community Based Participatory Action Research. Int J Nurs Educ Scholarsh, 16(1). doi:10.1515/ijnes-2018-0086

Beck, J., Greenwood, D. A., Blanton, L., Bollinger, S. T., Butcher, M. K., Condon, J. E., . . Wang, J. (2019). 2017 National Standards for Diabetes Self-Management Education and Support. Diabetes Educ, 45(1), 34-49. doi:10.1177/0145721718820941

Budig, K., Diez, J., Conde, P., Sastre, M., Hernan, M., \& Franco, M. (2018). Photovoice and empowerment: evaluating the transformative potential of a participatory action research project. BMC Public Health, 18(1), 432. doi:10.1186/s12889-018-5335-7

Canadian Council on Learning (CCL). (2008). Health literacy in Canada: A healthy understanding. Retrieved from: http://www.en.copian.ca/library/research/ccl/health/health.pdf 2008 [accessed 03. 06. 2020].

Canadian Public Health Association. (2014) Examples of health literacy in practice. Retrieved from: https://www.cpha.ca/sites/default/files/uploads/resources/healthlit/examples_e.pdf [accessed 03. 06. 2020].

Castellanos, D. C., \& Abrahamsen, K. (2014). Using the PRECEDE-PROCEED model to assess dietary needs in the Hispanic population in northeastern Pennsylvania. Hisp Health Care Int, 12(1), 43-53. doi:10.1891/1540-4153.12.1.43

Costa, I. G., Camargo-Plazas, P., \& Tregunno, D. (2017). Re-thinking self-care management for individuals with diabetic foot ulcers. Wounds Int $J$ 8(2), 10-14. 
Cramer, M. E., Lazoritz, S., Shaffer, K., Palm, D., \& Ford, A. L. (2018). Community Advisory Board Members' Perspectives Regarding Opportunities and Challenges of Research Collaboration. West $J$ Nurs Res, 40(7), 1032-1048. doi:10.1177/0193945917697229

Dawkins, V., \& Solomon, A. (2017). Introducing the World Cafe to Doctor of Nursing Practice Students. J Nurs Educ, 56(10), 638-639. doi:10.3928/01484834-20170918-11

Diabetes Canada Clinical Practice Guidelines Expert, C., Meneilly, G. S., Knip, A., Miller, D. B., Sherifali, D., Tessier, D., \& Zahedi, A. (2018). Diabetes in older people. Canadian Journal of Diabetes, 42 Suppl 1, S283-S295. doi:10.1016/j.jcjd.2017.10.021

Esienumoh, E. E., Allotey, J., \& Waterman, H. (2018). Empowering members of a rural southern community in Nigeria to plan to take action to prevent maternal mortality: A participatory action research project. $J$ Clin Nurs, 27(7-8), e1600-e1611. doi:10.1111/jocn.14244

Etowa, J. B., Bernard, W. T., Oyinsan, B., \& Clow, B. (2007). Participatory action research (PAR): an approach for improving black women's health in rural and remote communities. J Transcult Nurs, 18(4), 349-357. doi:10.1177/1043659607305195

Geboers, B., Brainard, J. S., Loke, Y. K., Jansen, C. J., Salter, C., Reijneveld, S. A., \& de Winter, A. F. (2015). The association of health literacy with adherence in older adults, and its role in interventions: a systematic meta-review. BMC Public Health, 15, 903. doi:10.1186/s12889-015-2251-y

Harris, A. N. (2019). Diabetes Self-management Education Provision by an Interprofessional Collaborative Practice Team: A Quality Improvement Project. Nurs Clin North Am, 54(1), 149-158. doi:10.1016/j.cnur.2018.11.002

IDF. (2019). IDF diabetes atlas Ninth edition. Retrieved from https://www.diabetesatlas.org/upload/resources/2019/IDF Atlas 9th Edition 2019.pdf

Johnson, S. M., Trejo, G., Beck, K. L., Worsley, C., Tranberg, H., Plax, K. L., \& Linton, J. M. (2018). Building Community Support Using a Modified World Cafe Method for Pregnant and Parenting Teenagers in Forsyth County, North Carolina. J Pediatr Adolesc Gynecol, 31(6), 614-619. doi:10.1016/j.jpag.2018.06.009

Kidd, S., Davidson, L., Frederick, T., \& Kral, M. J. (2018). Reflecting on Participatory, Action-Oriented Research Methods in Community Psychology: Progress, Problems, and Paths Forward. Am J Community Psychol, 61(1-2), 76-87. doi:10.1002/ajcp.12214

Loppie-Reading, C. \& Wien, F. (2009). Health inequalities and social determinants of Aboriginal peoples' health. National Collaborating Centre for Aboriginal Health. Prince George, BC: National Collaborating Centre for Aboriginal Health.

Newman, S. D. (2010). Evidence-based advocacy: Using photovoice to identify barriers and facilitators to community participation after spinal cord injury. Rehabilitation Nursing, 35(2), 47-59.

Nomura, Y., Matsuyama, T., Fukai, K., Okada, A., Ida, M., Yamauchi, N., . . Hanada, N. (2019). PRECEDEPROCEED model based questionnaire and saliva tests for oral health checkup in adult. J Oral Sci, 61(4), 544-548. doi:10.2334/josnusd.18-0288

O'Connor, M., \& Cotrel-Gibbons, L. (2017). World Cafe: a proactive approach to working with mentors. Nurs Manag (Harrow), 24(2), 26-29. doi:10.7748/nm.2017.e1553

Paredes-Chi, A. A., \& Castillo-Burguete, M. T. (2018). Is Participatory Action Research an innovative pedagogical alternative for training teachers as researchers? The training plan and evaluation for normal schools. Eval Program Plann, 68, 176-184. doi:10.1016/j.evalprogplan.2018.03.007 
Pinto, R. M., Spector, A. Y., Rahman, R., \& Gastolomendo, J. D. (2015). Research advisory board members' contributions and expectations in the USA. Health Promot Int, 30(2), 328-338. doi:10.1093/heapro/dat042

Rootman I, Gordon-El-Bihbety D. A vision for a health literate Canada: Report of the expert panel on health literacy. https://www.cpha.ca/sites/default/files/uploads/resources/healthlit/report_e.pdf 2008 [modified 06.16.2017].

Sezgin, D., \& Esin, M. N. (2018). Effects of a PRECEDE-PROCEED model based ergonomic risk management programme to reduce musculoskeletal symptoms of ICU nurses. Intensive Crit Care Nurs, 47, 89-97. doi:10.1016/j.iccn.2018.02.007

Shanta Bridges, L., Sharma, M., Lee, J. H. S., Bennett, R., Buxbaum, S. G., \& Reese-Smith, J. (2018). Using the PRECEDE-PROCEED model for an online peer-to-peer suicide prevention and awareness for depression (SPAD) intervention among African American college students: experimental study. Health Promot Perspect, 8(1), 15-24. doi:10.15171/hpp.2018.02

Twyman, S., Lehna, C., Fahey, E., Coty, M. B., \& Wishnia, G. (2015). Photovoice as a Way to Describe Home Fire Safety in a Group of Urban Seniors. Kentucky Nurse, 63(1), 12.

van Manen, M. (2014). Phenomenology of practice. meaning-giving methods in phenomenological research and writing. Walnut Creek, California. Left Coast Press.

WHO. (2019). Classification of diabetes mellitus 2019. Retrieved from https://apps.who.int/iris/handle/10665/325182 